\title{
Identification of potential therapeutic target genes in mouse mesangial cells associated with diabetic nephropathy using bioinformatics analysis
}

\author{
XIN MOU*, DI YI ZHOU*, YING HUI LIU, KAIYUAN LIU and DANYANG ZHOU \\ Department of Endocrinology, Zhejiang Integrated and Western Medicine Hospital, Hangzhou, Zhejiang 310003, P.R. China
}

Received September 11, 2018; Accepted April 9, 2019

DOI: $10.3892 /$ etm.2019.7524

\begin{abstract}
The aim of the present study was to identify genes under the effect of transforming growth factor- $\beta$ (TGF- $\beta 1$ ), high glucose (HG) and glucosamine $(\mathrm{GlcN})$ in MES-13 mesangial cells and elucidate the molecular mechanisms of diabetic nephropathy (DN). The gene expression datasets GSE2557 and GSE2558 were downloaded from the Gene Expression Omnibus database. Differentially expressed genes (DEGs) were independently screened using the GEO2R online tool. Gene Ontology and Kyoto Encyclopedia of Genes and Genomes (KEGG) pathway enrichment analyses were performed using the Database for Annotation, Visualization, and Integrated Discovery. The protein-protein interaction (PPI) network was constructed using the Search Tool for the Retrieval of Interacting Genes and Cytoscape software. The hub genes were identified by the NetworkAnalyzer plugin. Overlapping genes were subjected to molecular docking analysis using SystemsDock. A total of 202 upregulated and 158 downregulated DEGs from the HG-treated groups, 138 upregulated and 103 downregulated DEGs from the GlcN-treated groups, and 81 upregulated and 44 downregulated DEGs from the TGF- $\beta 1$-treated groups were identified. The majority of the DEGs were independently enriched in 'nucleosome assembly', 'chromatin silencing' and 'xenobiotic glucuronidation'. In addition, KEGG pathways were significantly enriched in 'systemic lupus erythematosus', 'protein processing in endoplasmic reticulum' and 'aldarate metabolism pathway', and 'TNF signaling pathway' intersected in the TGF- $\beta 1$-treated and HG-treated groups. In total, eight hub genes, Jun, prostaglandin-endoperoxide synthase 2 (Ptgs2), fibronectin 1 (Fn1), cyclin-dependent kinase (Cdk)2, Fos, heat
\end{abstract}

Correspondence to: Mrs. Danyang Zhou, Department of Endocrinology, Zhejiang Integrated and Western Medicine Hospital, 208 East Road, Hangzhou, Zhejiang 310003, P.R. China

E-mail: zdy0601@126.com

*Contributed equally

Key words: mesangial cells, diabetic nephropathies, differentially expressed genes, bioinformatics shock protein family A (Hsp70) member 5 (Hspa5), Hsp90b1 and homo sapiens hypoxia upregulated 1 (Hyou1), and three overlapping genes, Ras homolog gene family, member B (RHOB), complement factor $\mathrm{H}(\mathrm{CFH})$ and Krüppel-like factor 15 (KLF15), were selected. Valsartan with RHOB, and fosinopril with CFH and KLF15 had preferential binding activity. In conclusion, Jun, Ptgs2, Fn1, Cdk2, Fos, Hspa5, Hsp90b1, Hyou1, RHOB, CFH and KLF15 may be potential therapeutic targets for mesangial cells associated with DN, which may provide insight into DN treatment strategies.

\section{Introduction}

Diabetes mellitus (DM) is characterized by persistent hyperglycemia with an inability to fully utilize glucose and maintain insulin homeostasis, which leads to long-term damage and dysfunction of the eyes, kidneys, and nervous and cardiovascular systems in particular (1). Approximately $366,000,000$ individuals are expected to suffer from DM by the year 2030, and almost 592,000,000 individuals, or 1/10, are anticipated to have diabetes by $2035(1,2)$. It is estimated that $>40 \%$ of diabetic patients require regular, painful and expensive dialysis due to end-stage renal disease (ESRD), and the mortality rate remains high (3).

Diabetic nephropathy (DN) is an emerging clinical and public health challenge, which is also a life-threatening long-term microvascular complication of type 1 DM (T1DM) and type 2 DM (T2DM) $(1,4,5)$. Patients with DN are generally hypertensive, which increases the risk of cardiovascular disease and mortality (6); hypertension is interconnected with the initiation and progression of DN. The central pathological alterations during the initiation and progression of DN comprise mesangial extension, extracellular matrix alterations, tubulointerstitial fibrosis and glomerular sclerosis (7). In particular, alterations in the glomeruli, including fibrosis and apoptosis of mesangial cells, are relevant to the progression of DN. Evidence suggests that certain microRNAs (miRNAs) or transcription factors with abnormal expression may serve a vital role in the development of DN. Wu et al (8) reported that miRNA (miR)-455-3p suppresses renal fibrosis through repression of the expression of Rho-associated kinase 2 (ROCK2) in DN. miR-135a promotes renal fibrosis in DN by regulating transient receptor potential cation channel, subfamily C, member 1 (9), and inhibiting miR-192 ameliorates 
renal fibrosis in DN (10). Chen et al (11) reported that high glucose $(\mathrm{HG})$ promotes the expression of pro-inflammatory cytokines in vivo or in vitro, by upregulating high mobility group box 1 (HMGB1) and activating nuclear factor (NF)- $\mathrm{B}$ signaling in SV40 MES 13 cells. The condition of hyperglycemia also induces the expression of transforming growth factor- $\beta$ (TGF- $\beta$ ), fibronectin, collagen type IV and plasminogen activator inhibitor-1 (PAI-1), which are associated with fibrosis (12). Furthermore, increased renal mRNA and protein expression levels of TGF- $\beta 1$ have been observed in various animal models and in human DN (13). TGF- $\beta$, an important cytokine, is associated with the development of fibrosis and glomerulosclerosis, particularly mesangial cell phenotypic transformation in DN (14). Although considerable progress has been made towards elucidating the potential therapeutic targets and molecular mechanisms involved in DN, sufficient biomolecular target information and novel diagnostic and prognostic biomarkers that may be beneficial for improving the clinical management of DN appear to be lacking.

Gene expression profiling has been widely applied in clinical research as a potentially rapid and cost-effective approach, which is considered useful for identifying the expression levels of thousands of genes simultaneously. Furthermore, this method may provide a complete, systematic and reliable comparison of gene expression profiles among different tissue types. Accumulated core data of DN have been produced by gene chip analysis, and have been deposited into the Gene Expression Omnibus (GEO) database of the National Center for Biotechnology Information, a public database (http://www.ncbi.nlm.nih.gov/geo/). Therefore, the present study focused on extracting and reanalyzing the transcriptome profile of TGF- $\beta 1$-induced mesangial gene expression, and the difference between $\mathrm{HG}$ and glucosamine ( $\mathrm{GlcN})$-induced mouse mesangial cells. These data may assist in revealing the molecular mechanisms of DN in detail.

The main purpose of the present study was to identify the expression of differently expressed genes (DEGs) in a TGF- $\beta 1-$, HG-, and GlcN-induced mouse mesangial cell line (MES-13). A network pharmacology and bioinformatics reanalysis was performed of the original data (GSE2558 and GSE2557) of MES-13 cells from a study by Cheng et al (15), which were deposited in the GEO database. Subsequently, the DEGs between the TGF- $\beta 1, \mathrm{HG}, \mathrm{GlcN}$ and low glucose (LG) groups were screened via the application of GEO2R (http://www.ncbi.nlm.nih.gov/geo/geo2r/), with a cut-off value of $\mathrm{P}<0.05$ and $\mid \log 2^{\text {fold change }} \geq 1$ for statistical significance, followed by gene ontology $(\mathrm{GO})$ and Kyoto Encyclopedia of Genes and Genomes (KEGG) pathway enrichment analyses. Subsequently, the protein-protein interaction (PPI) network of the DEGs between the TGF- $\beta 1$, HG, GlcN and LG groups were respectively constructed via the Search Tool for the Retrieval of Interacting Genes (STRING; https://string-db. org/) database and visualized through Cytoscape software (http://www.cytoscape.org/), subsequently identifying hub genes in the PPI network with the highest values for degree, node betweenness and closeness via network topology calculation. Furthermore, computational docking was conducted to mimic the characteristics of anti-DN drug (captopril, enalapril, fosinopril, irbesartan, lisinopril, losartan, ramipril, telmisartan and valsartan in the DrugBank database)-target binding, using a web server for network pharmacology-based prediction and analysis, SystemsDock (http://systemsdock.unit. oist.jp/iddp/home/index). This present study applies network pharmacology and bioinformatics reanalysis to investigate the potential therapeutic target genes and pathways in TGF- $\beta 1$, $\mathrm{HG}$ and GlcN-induced mouse mesangial cells (MES-13). The results provide a comprehensive view of the associations and mechanisms underlying TGF- $\beta 1, \mathrm{HG}$ and GlcN-induced gene expression in renal mesangial cells at the molecular level, and identifies potential targets with the most appropriate drug for individualized anti-DN treatment.

\section{Materials and methods}

Microarray data. The gene expression profiles of GSE2558 and GSE2557 based on the platform of GPL1261 (Affymetrix Mouse Genome 4302.0 Array; Affymetrix, Inc., Santa Clara, CA, USA), and deposited by Cheng et al (15) from Wayne State University School of Medicine, which were downloaded from the GEO database of the National Center for Biotechnology Information (NCBI; http://www.ncbi.nlm. nih.gov/geo/). Renal mesangial cells serve an important role in the development of diabetic glomerulosclerosis and renal failure. The following procedures were deposited with the associated data sets and were previously published (15). The stable MES-13 murine mesangial cells transformed with non-capsid-forming SV-40 virus were obtained from the ATCC (Manassas, VA, USA) and exhibited a typical spindle-like appearance, positive staining for vimentin and desmin, and contraction in response to angiotensin II and expression of the AT1 receptor. The cells were maintained in DMEM and Ham's F-12 Nutrient Mixture (4:1 ratio; Gibco; Thermo Fisher Scientific, Inc., Waltham, MA, USA) containing a normal D-glucose concentration of $5.5 \mathrm{mmol} / 1$, $2 \%$ FCS (Gibco; Thermo Fisher Scientific, Inc.), $100 \mu \mathrm{g} / \mathrm{ml}$ streptomycin, $100 \mathrm{U} / \mathrm{ml}$ penicillin and $2 \mathrm{mmol} / 1$ glutamine, incubated in a humidified incubator with $5 \% \mathrm{CO}_{2}$ at $37^{\circ} \mathrm{C}$, and routinely passaged at confluence every 3 days by trypsinization using 10-cm culture dishes. Monolayers at 50\% confluence were starved in the above medium without FCS for 1 day and then incubated in starvation medium with $100 \mathrm{ng} / \mathrm{ml} \mathrm{TGF}-\beta 1$ for $24 \mathrm{~h}$ in GSE2558, containing four cell samples (GSM48386, GSM48392, GSM48394 and GSM4839), including the TGF- $\beta 1$-treated MES-13 mouse mesangial cell line and LG-treated cells as a control sample. For GSE2557, 50\% confluent monolayers were starved in the above medium without FCS for 1 day and incubated in the starvation medium with the desired concentrations of glucose and GlcN for $48 \mathrm{~h}$ (LG, $5.5 \mathrm{mM}$; HG, $25 \mathrm{mM}$; and $\mathrm{GlcN}$, $1.5 \mathrm{mM}+\mathrm{LG}$ ) in a humidified incubator containing $5 \% \mathrm{CO}_{2}$ at $37^{\circ} \mathrm{C}$, including two HG-treated cell samples (GSM48387 and GSM48388), two GlcN-treated cell samples (GSM48389 and GSM48390) and two LG-treated cell samples (GSM48385 and GSM48393) as the control cell samples.

Data processing and DEG identification. Comparisons were performed between the differently treated groups of samples (TGF- $\beta 1$-treated, vs. LG-treated cell samples in the GSE2558 dataset; HG-treated, vs. LG-treated cell samples and GlcN-treated, vs. LG-treated cell samples in the GSE2557 
dataset) to identify DEGs. The comparison was performed using the limma $\mathrm{R}$ package-based online program GEO2R (http://www.ncbi.nlm.nih.gov/geo/geo2r/). GEO2R allows the calculation and graphical representation as a box plot of the distribution of values for selected samples, and it applies an adjusted P-value using the Benjamini-Hochberg method to correct false-positives. Fold-changes (FC) in gene expression were calculated with threshold criteria of $\left|\log _{2}{ }^{\mathrm{FC}}\right| \geq 1$, and adjusted $\mathrm{P}<0.05$ was used as the cut-off criterion for statistically significant DEGs. Venny software (version 2.1; http://bioinfogp.cnb.csic.es/tools/venny/index.html) was used to analyze the overlapping DEGs in the three datasets.

Functional enrichment analysis. Gene Ontology (GO) analysis is a bioinformatics method for annotating genes and proteins to identify characteristic biological attributes, including biological process, cellular component, molecular function and biological functions, from high-throughput genome data $(16,17)$. The KEGG pathway database is a resource for understanding the high-level functions and utilities of a biological system, includes a variety of biochemical pathways (18). The Database for Annotation, Visualization, and Integrated Discovery (DAVID; version 6.7; https://david. ncifcrf.gov/), an online program that offers functional annotation of an enormous quantity of genes derived from various genomic resources, was used to perform GO and KEGG pathway analyses on the significant DEGs. The species was limited to Homo sapiens and $\mathrm{P}<0.05$ was considered to indicate a statistically significant difference.

PPI network construction and regulatory network analyses. The STRING database (version 10.0; http://string-db.org), which collects and predicts interaction information from genomic context predictions, high-throughput lab experiments, co-expression, automated text-mining and previous knowledge from databases (19) was used to predict the potential interactions between gene candidates at the protein level. Confidence scores $>0.4$ (median confidence score) were considered significant. The first three nodes and the overlapping DEGs in TGF- $\beta 1$-treated, HG-treated and GlcN-treated PPI networks were identified for further discussion and molecular docking. Additionally, Cytoscape software (version 3.4.0; http://www. cytoscape.org/) was utilized to construct the PPI network.

Molecular docking experiments based on SystemsDock. The DrugBank (www.drugbank.ca) is a unique, freely available bioinformatics and chemoinformatics resource containing information on drugs, drug-target interactions, drug actions and drug interactions, including Food and Drug Administration (FDA)-approved drugs in addition to experimental drugs undergoing the FDA approval process (20). The keyword 'Diabetic nephropathy' was input into the search field. Captopril, enalapril, fosinopril, irbesartan, lisinopril, losartan, ramipril, telmisartan and valsartan were selected as anti-DN drugs with different mechanisms for the molecular docking experiments. The overlapping DEGs for the crystal structures of Ras homolog gene family, member B (RHOB; PDB ID: 2FV8) and Krüppel-like factor 15 (KLF15; PDB ID: 4BN2) were obtained from the Protein Data Bank (http://www.rcsb.org/). The structure of complement factor $\mathrm{H}$ (CFH; PDB ID:3R62) was also obtained from the Protein Data Bank. SystemsDock (http://systemsdock.unit.oist.jp/iddp/home/index), a web server for network pharmacology-based prediction with high-precision docking simulation, was used to comprehensively characterize the ligand selectivity and to illustrate how a ligand acts on a complex molecular network (21). A docking score of $>4.25$ is considered fair, $5 \leq$ docking score $<7$ is considered good, and docking score $\geq 7$ is considered excellent; this scoring is conventionally used to classify ligand binding activity (22).

\section{Results}

Data processing and screening of differentially expressed mRNAs. The box plots of the mRNA expression levels were normalized to the same order of magnitude prior to the statistical analysis for GSE2557 and GSE2558, as presented in Fig. 1, which clearly demonstrated that the medians of the different samples were almost equivalent following normalization, indicating that the data were cross-comparable. According to the cut-off criteria of $\mathrm{P}<0.05$ and $\log _{2}{ }^{\mathrm{FC}} \mid>1.0$, a total of 360 DEGs were obtained, including 202 upregulated and 158 downregulated DEGs from the HG-treated groups compared with the LG-treated groups, and a total of 241 DEGs were obtained, including 138 upregulated and 103 downregulated DEGs from the GlcN-treated groups compared with the LG-treated groups, when redundant duplicate content had been removed from GSE2557. Furthermore, via integrated analysis, a set of 125 DEGs were identified in the TGF- $\beta 1$-treated groups compared with the LG-treated groups, including 81 upregulated and 44 downregulated DEGs in GSE2558.

Functional enrichment analysis. The GO enrichment results demonstrated that the significantly enriched GO terms of the DEGs in GSE2558 for biological process were 'xenobiotic glucuronidation', 'flavonoid glucuronidation', 'flavonoid biosynthetic process' and 'positive regulation of cell proliferation', whereas the significantly enriched GO terms for cellular component were 'extracellular space' and 'extracellular region'. Furthermore, the significantly enriched GO terms for molecular functions were 'glucuronosyltransferase activity', 'protein heterodimerization activity' and 'protein binding' (Fig. 2). In addition, the DEGs of the HG-treated groups compared with the LG-treated groups were mainly enriched in the GO biological process terms of "nucleosome assembly', 'negative regulation of megakaryocyte differentiation' and 'DNA replication-independent nucleosome assembly', 'cellular component terms of nucleosome', 'nuclear chromosome' and 'extracellular exosome', and molecular functions terms of 'DNA binding', 'histone binding' and 'protein domain specific binding' (Fig. 3). The significantly enriched GO biological process terms of 'chromatin silencing', 'response to endoplasmic reticulum stress' and 'endoplasmic reticulum unfolded protein response', the GO terms of cellular component 'endoplasmic reticulum', 'endoplasmic reticulum chaperone complex' and 'endoplasmic reticulum lumen', and the GO terms of molecular function 'oxidoreductase activity' and 'misfolded protein binding' were noted for DEGs of the GlcN-treated groups compared with the LG-treated groups (Fig. 4). 

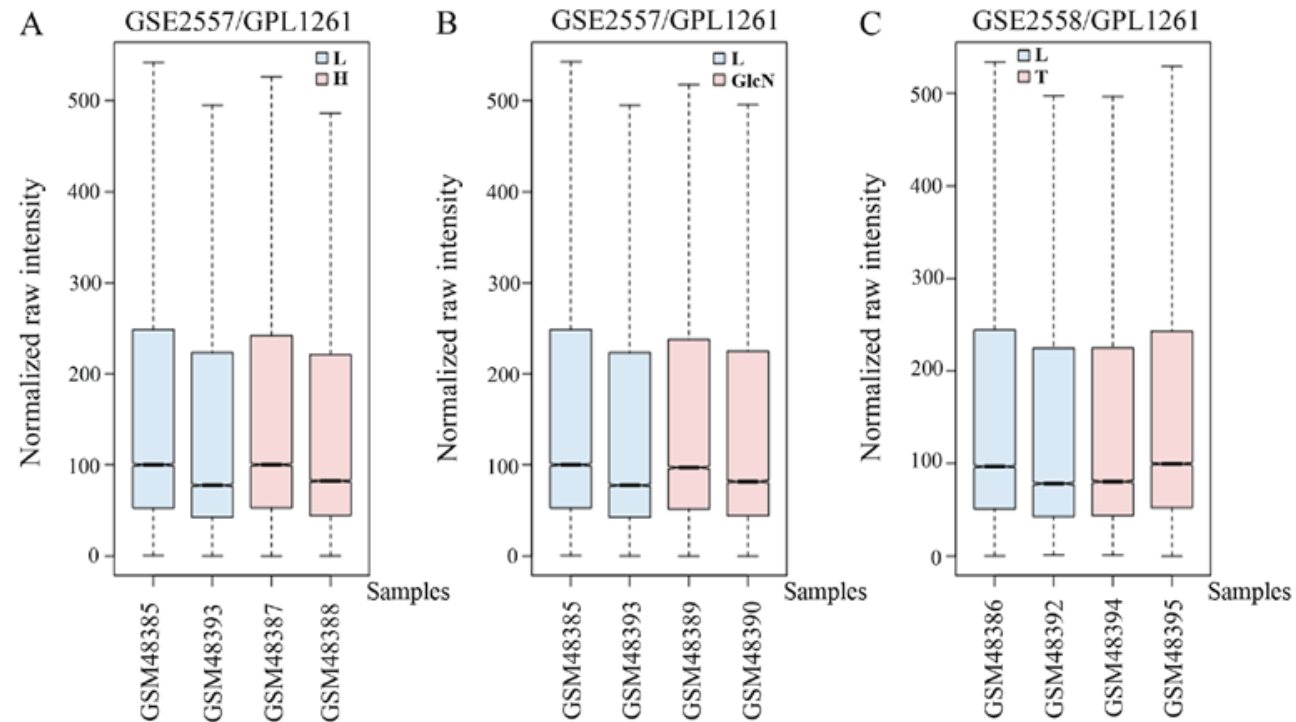

Figure 1. Box plots for the GSE2557 and GSE2558 datasets following normalization. The x-axis represents the samples and the y-axis represents the distribution of expression levels. (A) Distribution of gene expression levels in the $\mathrm{H}$ and $\mathrm{L}$ groups; (B) distribution of gene expression levels in the GlcN and L groups; (C) distribution of gene expression levels in the T and L groups. $\mathrm{H}$, high glucose; $\mathrm{L}$, low glucose; GlcN, glucosamine; T, transforming growth factor- $\beta$.

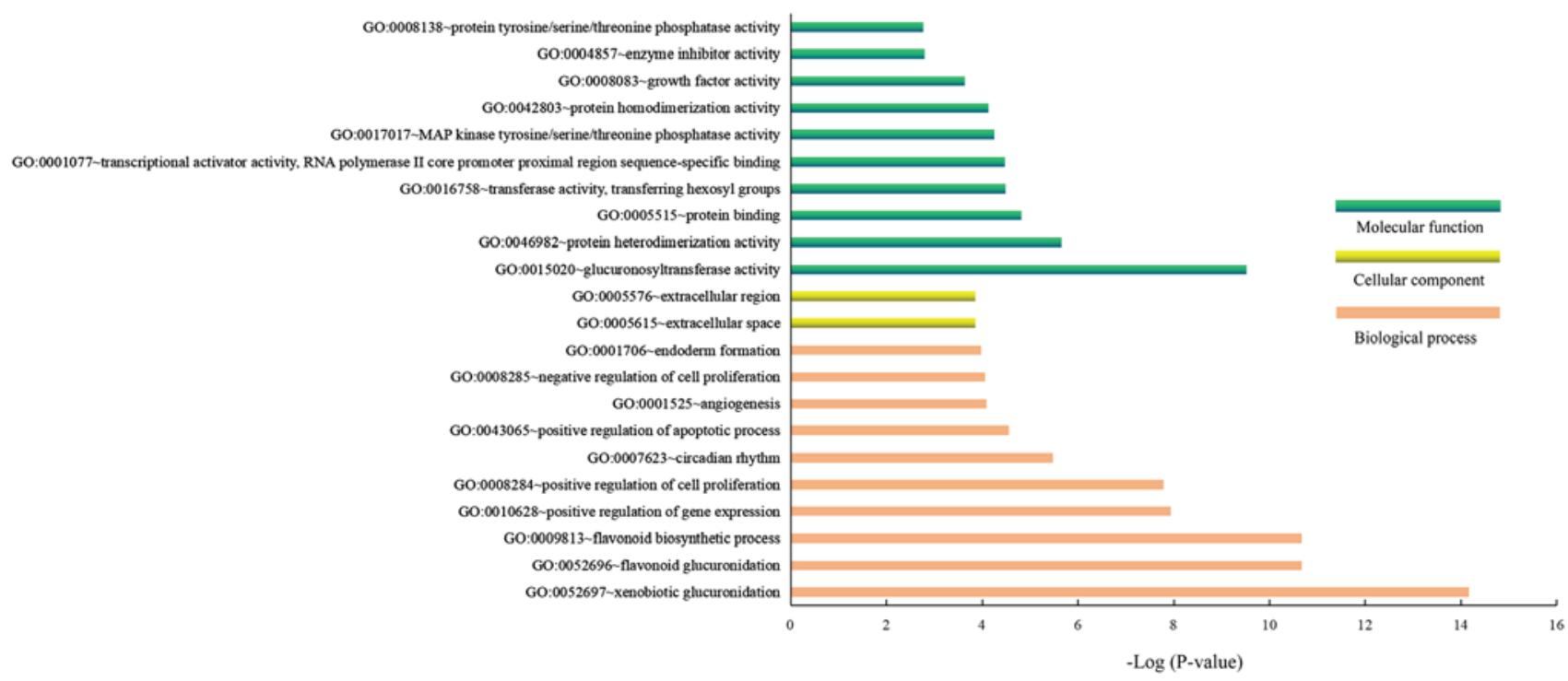

Figure 2. GO analysis of the differentially expressed genes following treatment with transforming growth factor- $\beta 1$. Green represents molecular function; yellow represents cellular component; orange represents biological process. The-log P-value is shown on the x-axis. GO, Gene Ontology.

Furthermore, the KEGG pathway analysis indicated that the 'protein processing in endoplasmic reticulum', 'biosynthesis of antibiotics' and 'systemic lupus erythematosus' pathways served an essential role in the GlcN-treated groups compared with the LG-treated groups (Fig. 5A). 'Systemic lupus erythematosus', 'alcoholism' and 'Viral carcinogenesis' were the top significantly enriched pathways in the HG-treated groups compared with the LG-treated groups (Fig. 5B). As presented in Fig. 5C. The DEGs of the TGF- 31 -treated groups compared with the LG-treated groups were primarily enriched in 'ascorbate and aldarate metabolism', 'pentose and glucuronate interconversions' and 'porphyrin and chlorophyll metabolism'. Notably, the intersecting pathway was 'TNF signaling pathway' in the TGF- $\beta 1$-treated and HG-treated groups, compared with the LG-treated groups.
PPI network. Based on the information obtained from the STRING database, a network diagram was produced separately for significant DEGs in GSE2557 and GSE2558; certain DEGs were on the margins and isolated, suggesting no association with other genes, and were removed from the PPI network diagram using Cytoscape. There were 64 nodes and 149 edges in the network for the TGF- $\beta 1$-treated groups compared with the LG-treated groups (Fig. 6A), 223 nodes with 1,245 edges in the network for the HG-treated groups compared with the LG-treated groups (Fig. 6B), and 134 nodes with 366 edges in the network for the GlcN-treated groups compared with the LG-treated groups (Fig. 6C). In a PPI network, the greater the characteristic properties of degree, betweenness and closeness of a node, the more important its role. The parameters 'degree', 'betweenness' and 'closeness' were used to select the 


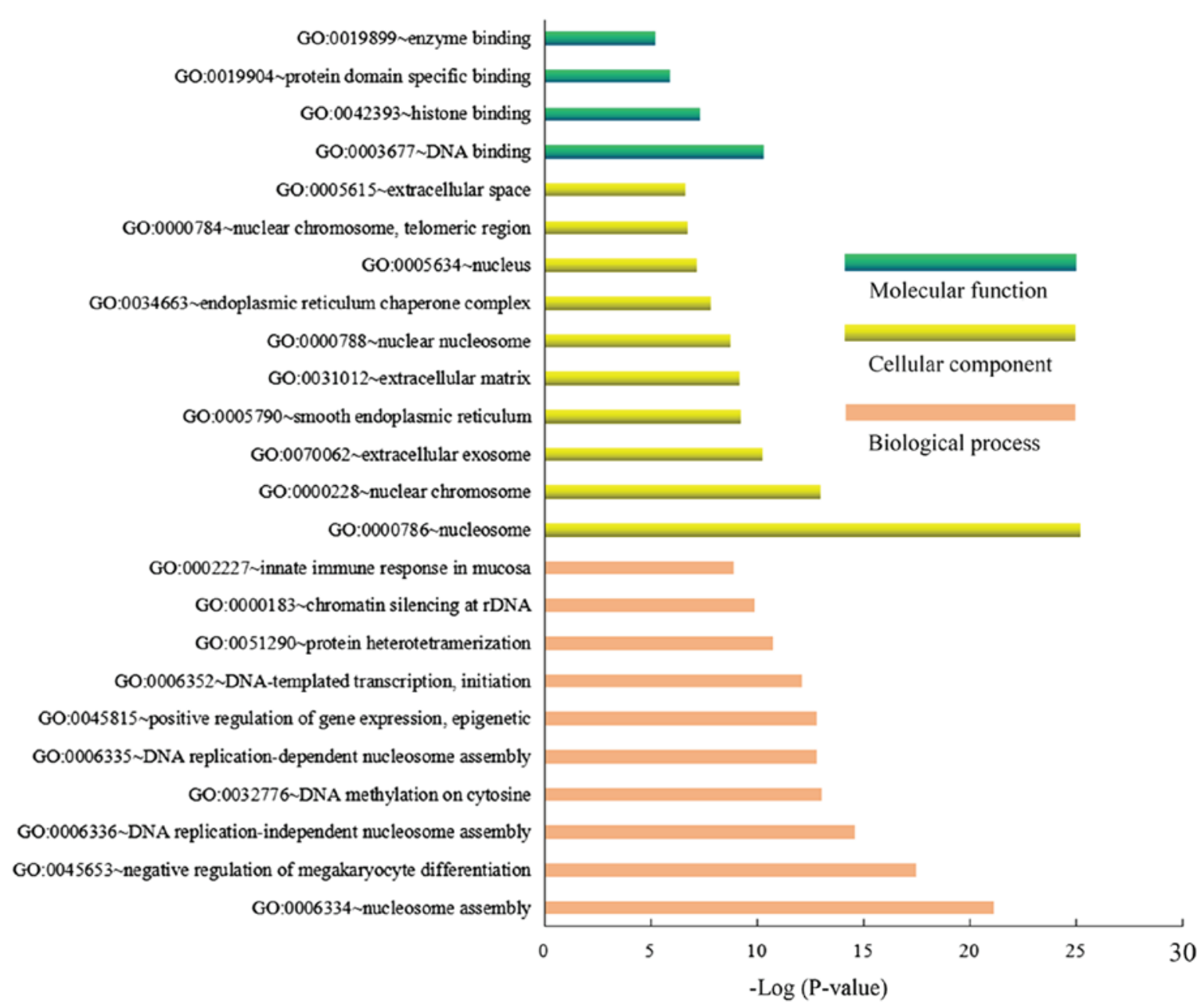

Figure 3. GO analysis of the differentially expressed genes following treatment with high glucose. Green represents molecular function; yellow represents cellular component; orange represents biological process. The-log P-value is shown on the x-axis. GO, Gene Ontology.

first three nodes, Jun (degree $=32$, betweenness $=0.49093999$, closeness $=0.68292683)$, prostaglandin-endoperoxide synthase 2 (Ptgs2; degree $=17$, betweenness $=0.0727469$, closeness $=0.52336449)$ and fibronectin $1(\mathrm{Fn} 1$; degree $=16$, betweenness $=0.18112793$, closeness $=0.55445545)$ in Fig. 4A; Jun (degree $=56$, betweenness $=0.25066792$, closeness $=0.51318945)$, cyclin-dependent kinase $(\mathrm{Cdk} 2$; degree $=43$, betweenness $=0.14884068$, closeness $=0.49195402)$ and Fos (degree $=41$, betweenness $=0.08047788$, closeness $=0.47240618)$ in Fig. 4B; heat shock protein family A (Hsp70) member (Hspa)5 (degree $=30$, betweenness $=0.38665873$, closeness $=0.42635659$ ), Hsp90b1 (degree=21, betweenness $=0.07358151$, closeness $=0.38327526$ ) and Hyou1 (degree $=17$, betweenness $=0.01014303$, closeness $=0.34920635)$ in Fig. 4 C. These genes were assessed as hub genes.

Finally, to improve the specificity, a total of two upregulated potentially interacting genes (Rhob and Cfh) in the Venn diagram (Fig. 7A), and one downregulated potential therapeutic target gene (Klf15) (Fig. 7B) in the three sets of results were identified and selected for further investigation.

Molecular docking analysis of binding to RHOB, CFH and $K L F 15$. Computational molecular docking experiments were conducted to mimic the characteristics of anti-hypertension or DN-target binding. The crystal structure of RHOB (PDB
ID: 2FV8), KLF15 (PDB ID: 4BN2) and the structure of CFH (PDB ID:3R62) were obtained from the Protein Data Bank (http://www.rcsb.org/). The docking exercise was conducted using systemsDock (http://systemsdock.unit.oist. jp/iddp/home/index) (21). The results indicated that a number of the selected drugs, lisinopril, enalapril, ramipril, fosinopril and valsartan, in the drugbank database had higher docking scores, of 5.727, 5.936, 6.19, 6.415 and 6.692 respectively, compared with the native ligand (5.7) of RHOB (Fig. 8A). As presented in Fig. 8B, the binding scores of irbesartan, captopril, losartan, lisinopril, valsartan, enalapril, ramipril and fosinopril were 3.904, 4.409, 4.836, 5.749, 5.924, 5.957, 6.231 and 6.464, respectively, which were higher than that of the native ligand (3.7) of CFH. Similarly, the binding scores of lisinopril, enalapril, ramipril, valsartan and fosinopril were 5.717, 5.760, 5.844, 6.248 and 6.614, respectively, which were higher than the score of 5.70 of the natural ligand of KLF15 (Fig. 8C). The results of the molecular docking of binding to RHOB, CFH and KLF15 indicted that valsartan, fosinopril, or a combination of the two may have a superior therapeutic effect via the putative targets.

\section{Discussion}

DN, a common microvascular complication of diabetes, and a major cause of ESRD, a leading cause of mortality among 


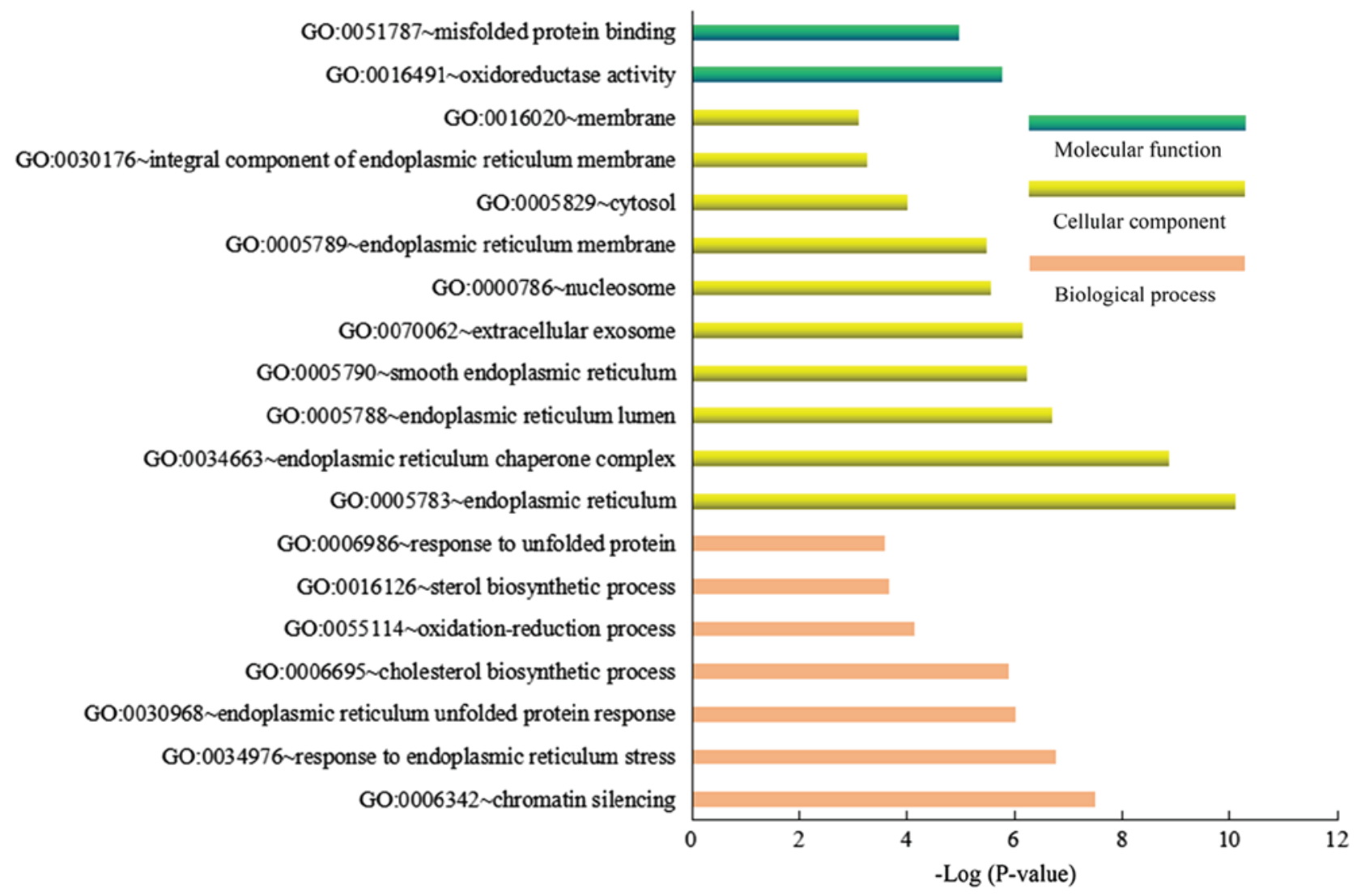

Figure 4. GO analysis of the differentially expressed genes following treatment with glucosamine. Green represents molecular function; yellow represents cellular component; orange represents biological process. The-log P-value is shown on the x-axis. GO, Gene Ontology.

diabetic patients $(23,24)$. It is characterized by proteinuria, albuminuria, renal glomerular hypertrophy, basement membrane thickening, podocyte dysfunction, and mesangial and tubulointerstitial fibrosis due to accumulation of extracellular matrix (ECM) proteins (25-27). Previous investigations have demonstrated that one of the principal classical pathways leading to renal fibrosis is the TGF- $\beta 1 /$ Smad signaling pathway, and culturing mesangial cells, the culprit cells in DN, in HG media stimulates the synthesis of ECM proteins, inhibits cell growth and alters the production of growth factors (15). TGF- $\beta 1$ is one of the most important mediators of fibrosis, in addition to cellular hypertrophy and survival $(26,28)$. Following TGF- $\beta 1 /$ Smad signaling pathway activation, the expression of TGF- $\beta 1$ is increased, inducing excessive deposition of ECM and increased epithelial-mesenchymal transition in renal cells, including mesangial cells and tubular cells in DN, indicating that TGF- $\beta 1$ serves a critical role in the development and progression of fibrosis in DN. Comprehensive investigations to improve current understanding of the molecular mechanisms of the pathogenesis of DN are thus important and necessary.

In the present study, putative hub candidate genes and enriched pathways of TGF- $\beta 1$, HG-, and GlcN-stimulated murine mesangial (MES-13) cells were identified by bioinformatics analysis and the verification of hub genes was performed via molecular docking. The gene expression data from GSE2557 and GSE2558 were reanalyzed and identified. A total of 360 DEGs were screened, consisting of 202 upregulated and 158 downregulated DEGs from the HG-treated groups compared with the LG-treated groups; in addition,
241 DEGs were obtained, including 138 upregulated and 103 downregulated DEGs from the GlcN-treated groups compared with the LG-treated groups in GSE2557, and 125 DEGs were identified, including 81 upregulated and 44 downregulated DEGs, in GSE2558. The present study identified three overlapping DEGs, two upregulated and one downregulated, across the three conditions. Furthermore, the results of the GO analysis indicated that the DEGs of the TGF- $\beta 1$-treated groups compared with those in the LG-treated groups were primarily involved in the 'xenobiotic glucuronidation', 'flavonoid glucuronidation', 'flavonoid biosynthetic process' and 'positive regulation of cell proliferation', and the 'ascorbate and aldarate metabolism' pathways; the DEGs of the HG-treated groups compared with the LG-treated groups were principally enriched in the 'nucleosome assembly', 'negative regulation of megakaryocyte differentiation' and the 'systemic lupus erythematosus' pathways; and the DEGs of the GlcN-treated groups compared with the LG-treated groups were involved in the 'chromatin silencing', 'response to endoplasmic reticulum stress', 'endoplasmic reticulum unfolded protein response' and the 'protein processing in endoplasmic reticulum' pathways. In addition, KEGG pathway enrichment analysis demonstrated that the overlapping pathway was the 'TNF signaling pathway' in the TGF- $\beta 1$-treated and HG-treated groups compared with the LG-treated groups. There is evidence to suggest that oxidative stress, inflammation and fibrosis serve a pivotal role in the progression of DN. Based on a number of human and animal studies, tumor necrosis factor- $\alpha$ (TNF- $\alpha$ ), interleukin-1 $\beta$ (IL-1 $\beta$ ) and interleukin-6 (IL-6), in addition 
A Pathways of TGF- $\beta 1$

mmu05034:Alcoholism

mmu05322: Systemic lupus erythematosus

mmu01130:Biosynthesis of antibiotics

mmu04141:Protein processing in endoplasmic reticulum

B Pathways of HG

mmu04668:TNF signaling pathway

mmu04141:Protein processing in endoplasmic reticulum

mmu05203:Viral carcinogenesis

mmu05034:Alcoholism

mmu05322: Systemic lupus erythematosus

C Pathways of GlcN

mmu04668:TNF signaling pathway mmu04010:MAPK signaling pathway mmu00830: Retinol metabolism mmu00140:Steroid hormone biosynthesis mmu00983:Drug metabolism - other enzymes mmu00982:Drug metabolism - cytochrome P450 mmu05204:Chemical carcinogenesis mmu00980: Metabolism of xenobiotics by cytochrome P450 mmu00860:Porphyrin and chlorophyll metabolism mmu00040:Pentose and glucuronate interconversions mmu00053:Ascorbate and aldarate metabolism

\begin{tabular}{llccccc}
0 & 5 & 10 & 15 & 20 & 25 & 30 \\
& \multicolumn{4}{c}{$\begin{array}{c}\text {-Log (P-value) } \\
\end{array}$} &
\end{tabular}

Figure 5. Kyoto Encyclopedia of Genes and Genomes pathway analysis of differentially expressed genes following treatment with TGF- $\beta 1$, HG and GlcN (A) Treatment with TGF- $\beta 1$ (red); (B) treatment with HG (black); (C) treatment with GlcN (green). HG, high glucose; GlcN, glucosamine; TGF- $\beta 1$, transforming growth factor $\beta$.

to the expression of numerous pro-inflammatory cytokines and chemokines, are increased in DN with the activation of the $\mathrm{NF}-\kappa \mathrm{B}$ pathway in the kidneys of diabetic patients, which in turn activates the TGF- $\beta 1 /$ Smad pathway to promote the progression of fibrosis (29-31). Notably, TGF- $\beta 1$ may be acting through the TNF signaling pathway and worsening inflammatory responses in DN. These enriched GO terms and pathways provide insights into the molecular mechanism of $\mathrm{DN}$, and may therefore be useful for the development of novel therapeutic strategies.

In the present study, DEGs were screened from the GES2557 and GSE2558 microarray datasets. Jun, Ptgs2 and Fn1 were identified as hub genes under treatment with TGF- $\beta 1$, and Cdk2, Fos, Hspa5, Hsp90b1 and Hyou1 also were selected as hub genes. A number of these genes have been linked to DN in previous studies. Jun is one of the components of the nuclear transcription factor activator protein-1 (AP-1), and binds to TRE elements in target gene promoter regions in order to activate transcription (32). Weigert et al (33) revealed that the mutation of Jun binding sites in the TGF $\beta 1$ promoter or treatment with a Jun inhibitor eliminates HG-induced TGF $\beta 1$ promoter activity in mesangial cells. Similarly, Gao et al (34) suggested that the increase in the expression of Jun and SP1 is synergistically activated, and is positively correlated with profibrotic expression of TGF $\beta 1$ in HG-treated human renal mesangial cells and in the development of DN by autoregulation, cross-activation and phospho-modification. PTGS, also known as cyclooxygenase (COX), consists of two major isoforms, Ptgs1 (COX-1) and Ptgs2 (COX-2). The cyclooxygenase/prostaglandin system contributes to the development of $\mathrm{DN}$ (35). COX-1 is expressed constitutively in numerous tissues, whereas COX-2 is expressed in various organs during inflammation and is expressed at high levels in podocytes, mesangial cells and macula densa cells in diabetic rats (36). Cheng et al (37) and Cheng et al (38) 
A

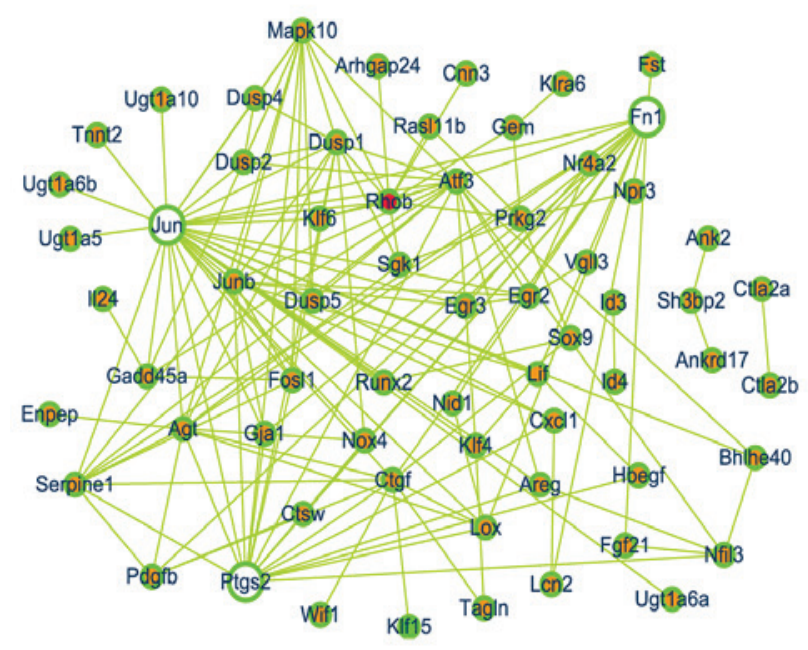

B

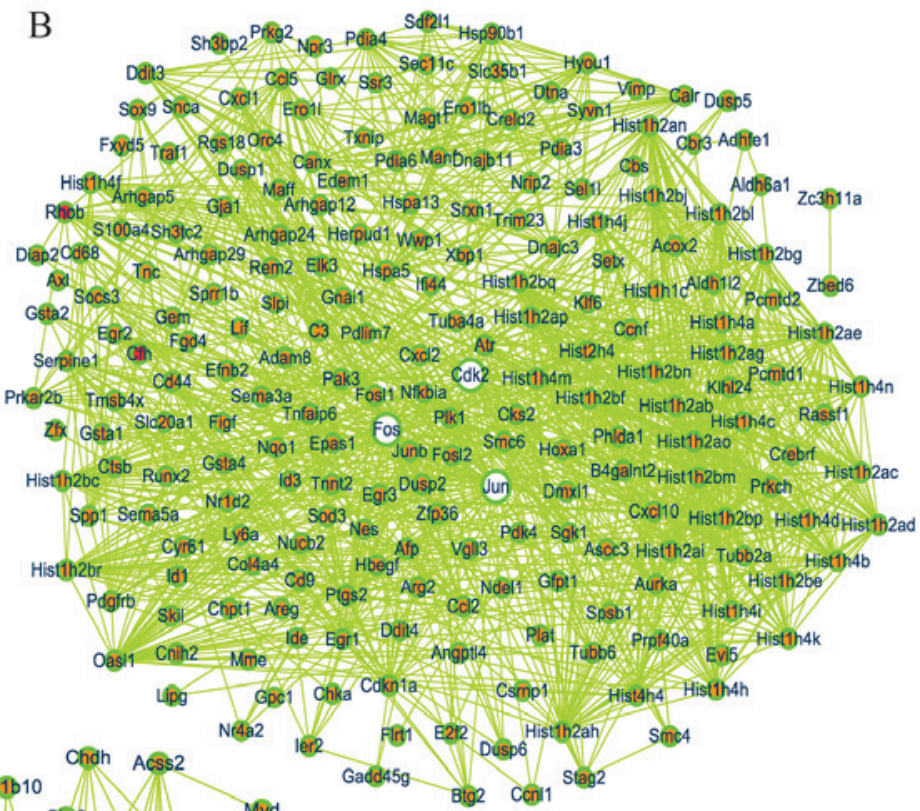

$\mathrm{C}$

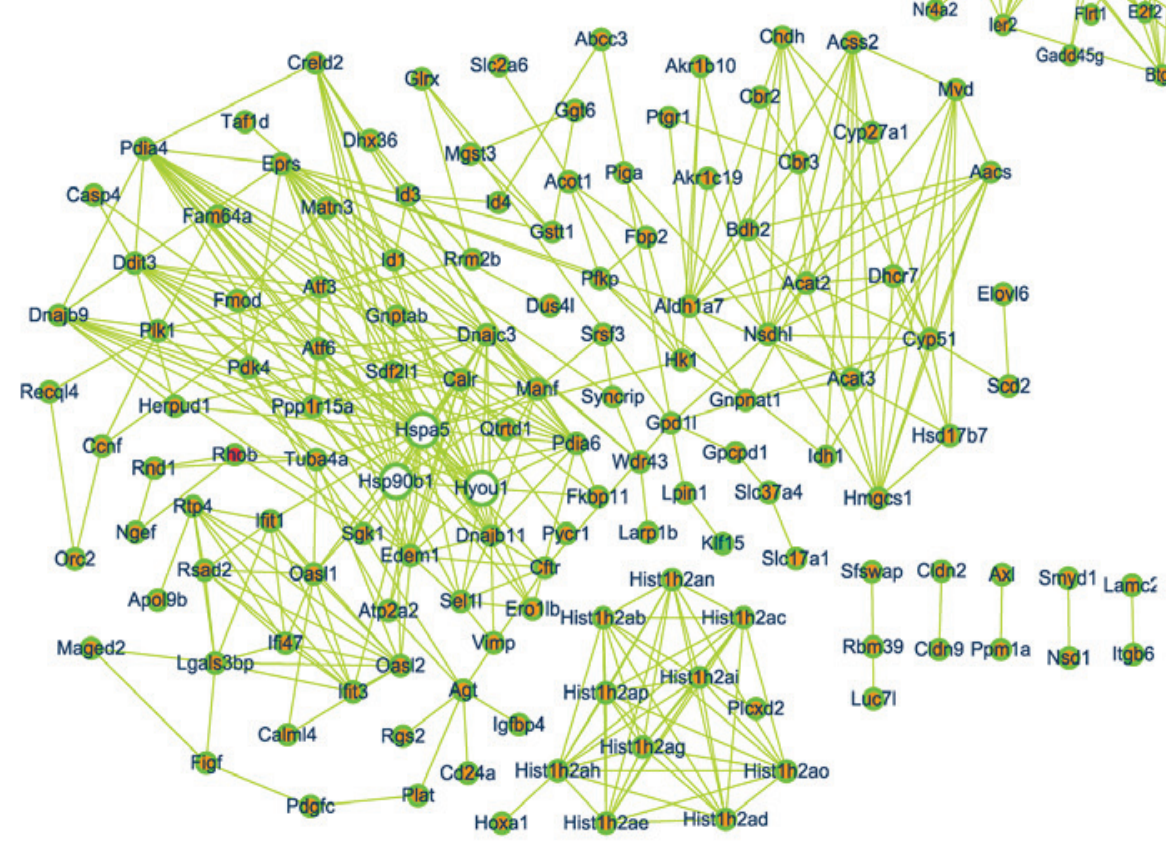

Figure 6. PPI network construction for differentially expressed genes. (A) Treatment with transforming growth factor- $\beta 1$; (B) treatment with high glucose; (C) treatment with glucosamine. The filled white, orange and green nodes in the PPI networks were selected as hub genes. The filled orange nodes represent upregulated and green nodes represent downregulated. Green lines represent interaction between nodes. PPI, protein-protein interaction.

reported that DN increases podocyte expression of $\mathrm{COX}-2$, and the inhibition of COX-2 reduces proteinuria and glomerular injury and delays DN progression in animal models of diabetes. ECM accumulation may induce further tubular endothelium fibrosis and glomerular sclerosis and cause the glomerular filtration rate to progressively decrease, leading to renal failure in DN. Fn1, a noncollagenous glycoprotein and the principal component of ECM, is increased substantially in DN and leads to glomerular sclerosis and eventual fibrosis (39). CDKs serve key roles in cell proliferation, and Cdk2 is a member of the CDK family, which is considered to be essential in the cell cycle and associates with cyclin A, driving cells through the $\mathrm{S}$ phase (40). Saurus et al (41) provided a novel strategy to prevent podocyte apoptosis and the progression of DN but preventing the downregulation of $\mathrm{Cdk} 2$, by inhibiting the Toll-like receptor (TLR) pathway with 4,5-dihydro-3-phenyl-5-isoxazoleacetic acid. The Fos (c-Fos, FosB, Fra-1 and Fra-2) and Jun (c-Jun,
JunB and JunD) proteins form dimers with AP-1, which serve an important role in DN (42). In the present study, the expression of Fos was significantly upregulated, which was consistent with the activation of AP-1 by HG (42). The activation of AP-1 induced by hyperglycemia, oxidative stress, advanced glycation-end products, low-density lipoprotein, angiotensin II and proinflammatory cytokines under diabetic conditions may regulate the expression of FN, TGF- $\beta 1$ and intercellular adhesion molecule, indicating that AP-1 is closely correlated with inflammation, cell proliferation and ECM accumulation during the progression of DN (42-44). Endoplasmic reticulum (ER) stress, resulting from the accumulation of misfolded and/or unfolded proteins in ER membranes, and induced podocyte apoptosis serve a critical role in the development of DN $(45,46)$. Hspa5, a member of the HSP70 family, is also known as glucose-regulated protein 78 (GRP78/Bip), an important molecular chaperone localized to the ER, and has 

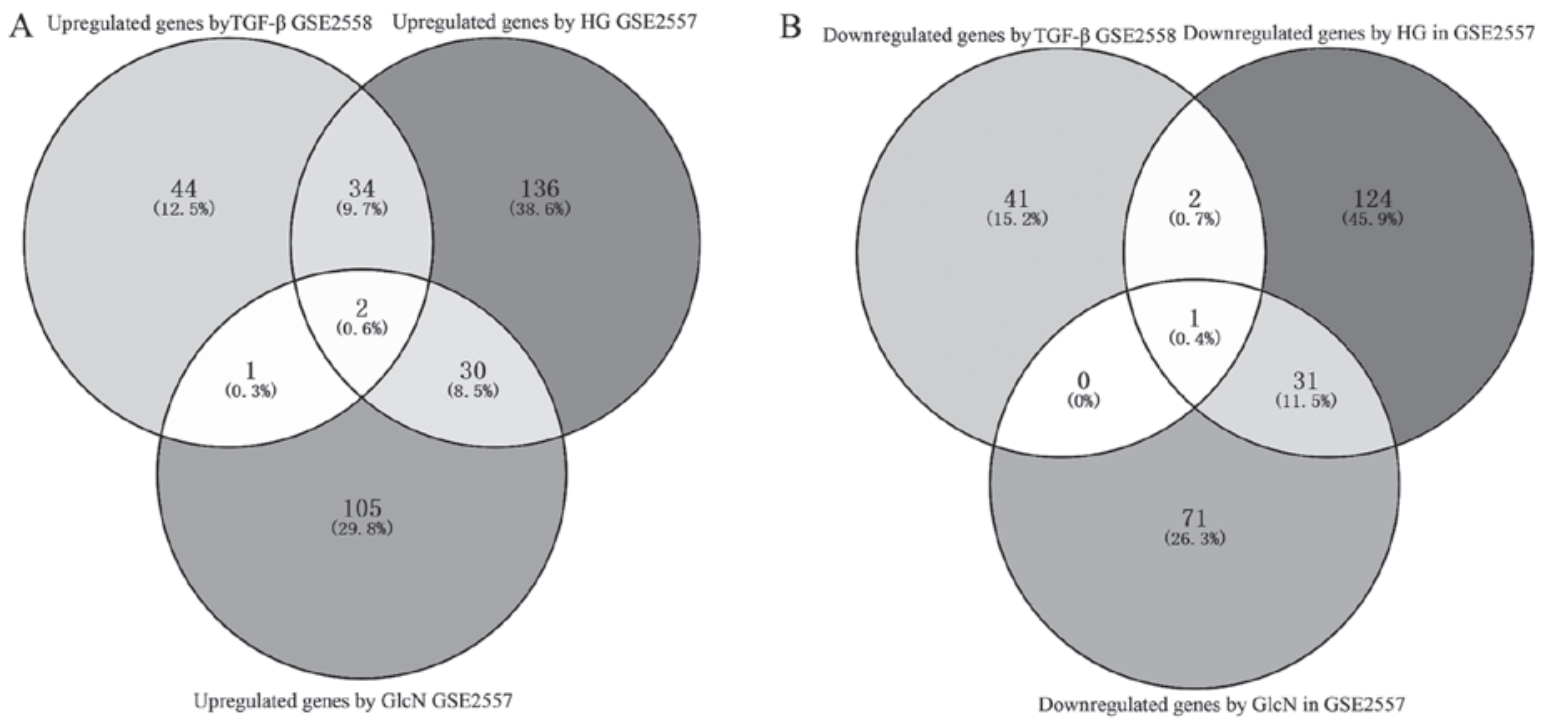

Figure 7. Gene co-regulation by TGF- $\beta 1, \mathrm{HG}$ and GlcN in mesangial cells. (A) Two upregulated overlapping genes (Ras homolog gene family, member B and complement factor H). (B) One downregulated overlapping gene (Krüppel-like factor 1). HG, high glucose; GlcN, glucosamine; TGF- $\beta 1$, transforming growth factor- $\beta$.
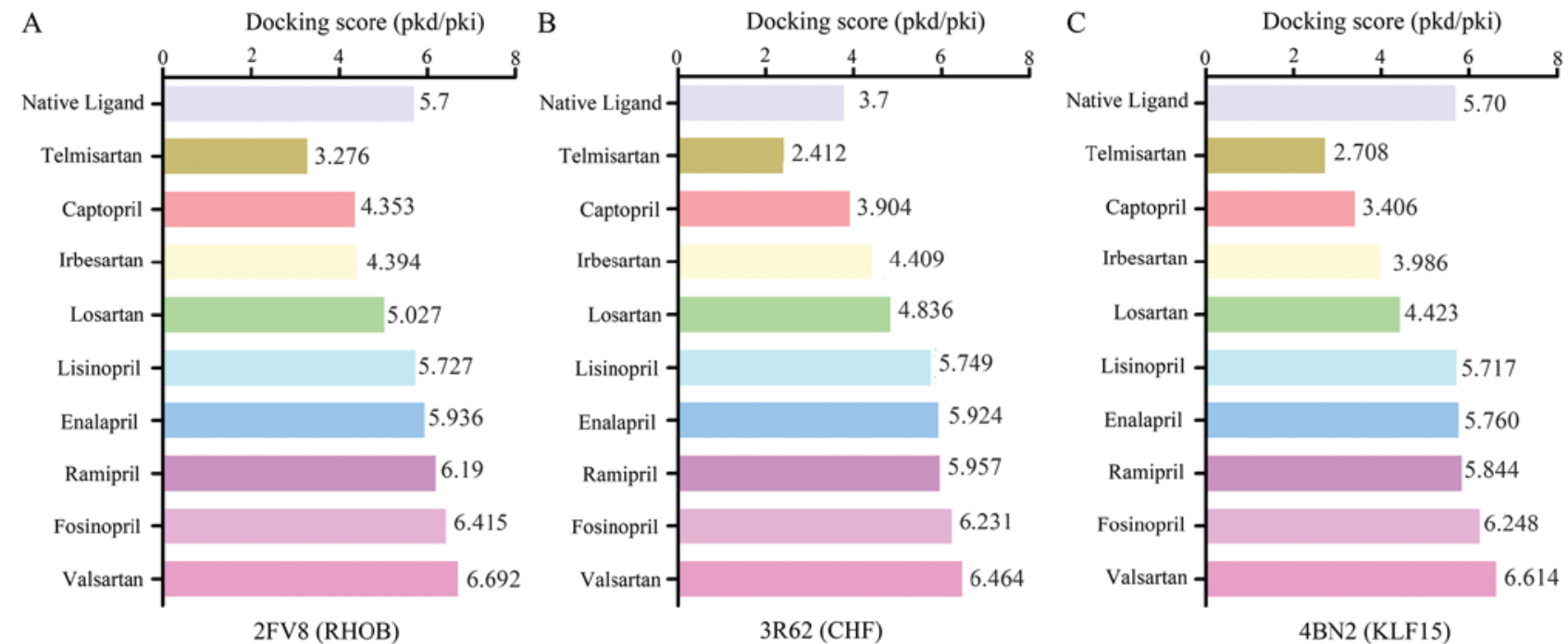

Figure 8. Molecular docking of binding to RHOB, CFH and KLF15. The y-axis represents captopril, enalapril, fosinopril, irbesartan, lisinopril, losartan, ramipril, telmisartan and valsartan as antidiabetic nephropathy drugs, and the $\mathrm{x}$-axis represents the docking score values. The docking score of these drugs binding to (A) RHOB, (B) CFH and (C) KLF15 are presented. Selected drugs, lisinopril, enalapril, ramipril, fosinopril and valsartan, exhibited similar or superior docking than the native ligands. RHOB, Ras homolog gene family, member B; CFH, complement factor H; KLF15, Krüppel-like factor 1.

been extensively considered as an important indicator for the induction of ER stress; the upregulated expression of GRP78 also indicates the activation of ER stress (46). Hyou1 also belongs to the ER chaperone family. Lindenmeyer et al (47) reported that patients with established DN had a marked increase in the mRNA expression of the genes Hspa5 and Hyou1. Cao et al (45) and Chen et al (48) respectively reported that the ER stress inhibitors ursodeoxycholic acid and 4-phenylbutyrate and terpene glycoside component from Moutan Cortex ameliorate DN by regulating ER stress-associated inflammatory responses, as evidenced by the decreased expression of GRP78/Bip. Hsp90b1 encodes a member of a family of adenosine triphosphate-metabolizing molecular chaperones with roles in stabilizing and folding other proteins, also known as GRP94. GRP94 is the most abundant protein contributing to ER quality control by chaperoning the folding of proteins, participating in calcium storage and assisting in the targeting of misfolded proteins for ER-associated degradation in the ER lumen (49). Based on the results from GSE2557 in the present study, GlcN may downregulate the expression of GRP94 in MES-13 cells.

The results of the molecular docking demonstrated that valsartan with the overlapping gene RHOB, and fosinopril with $\mathrm{CFH}$ and KLF15 had preferential binding activity. RHOB is an early-response gene whose expression is elevated by cellular stresses, and which serves an important role in the onset of DM and its associated complications (50). Bravo-Nuevo et al (51) revealed that RHOB loss prevents streptozotocin-induced 
diabetes and ameliorates diabetic complications in mice. The complement system is a key component of innate immunity. $\mathrm{CFH}$ is a $155-\mathrm{kDa}$ glycoprotein and consists of 20 short census repeat domains. It is located on chromosome 1q32 and encodes the factor $\mathrm{H}$ protein, a critical inhibitor of the alternative pathway of complement in the fluid phase and on the surface of host cells $(52,53)$. Bonomo et al (53) indicated that a subset of cases with end-stage kidney disease clinically ascribed to the effects of hypertension or glomerulosclerosis actually have $\mathrm{CFH}$-associated forms of mesangial proliferative glomerulonephritis. Podocyte injury is the hallmark of proteinuric kidney disease. The kidney-enriched zinc finger transcription factor KLF15 belongs to a 17-member family of DNA-binding zinc finger transcription factors associated with differentiation, mitochondrial biogenesis, and cell cycle and DNA repair, a diverse set of cellular processes (54). Furthermore, previous reports have suggested that inducing podocyte-specific KLF15 attenuates kidney injury by directly and indirectly upregulating genes critical for podocyte differentiation, suggesting that KLF15 induction may be a potential strategy for treating proteinuric kidney disease (55).

In conclusion, this comprehensive bioinformatics analysis identified that a total of 11 hub genes, including Jun, Ptgs2, Fn1, Cdk2, Fos, Hspa5, Hsp90b1, Hyou1, RHOB, CFH and KLF15, and the TNF signaling pathway in the MES-13 mouse mesangial cell line may serve a vital role in the progression of DN. These findings provide a set of useful molecular targets for future investigation of the mechanisms and selection of biotargets for DN. Further experimental investigations or the use of other datasets are to be performed to confirm the function of the identified genes in the present observations.

\section{Acknowledgements}

Not applicable.

\section{Funding}

This study was supported by grants from the National Natural Science Foundation of China (grant nos. 81774217 and 81273623), a grant from Zhejiang Administration of Traditional Chinese Medicine (grant no. 2017ZKL016), and a grant from the Science and Technology Commission of Hangzhou (grant no. 20150733Q42).

\section{Availability of data and materials}

The analyzed data sets generated during the present study are available from the corresponding author on reasonable request.

\section{Authors' contributions}

XM, DiYZ and DaYZ collected and analyzed the majority of the data and drafted the initial manuscript. YHL and KYL collected important background information. All authors read and approved the final manuscript.

\section{Ethics approval and consent to participate}

Not applicable.

\section{Patient consent for publication}

Not applicable.

\section{Competing interests}

The authors declare that they have no competing interests.

\section{References}

1. Sathibabu Uddandrao VV, Brahmanaidu P, Ravindarnaik R, Suresh P, Vadivukkarasi S and Saravanan G: Restorative potentiality of S-allylcysteine against diabetic nephropathy through attenuation of oxidative stress and inflammation in streptozotocin-nicotinamide-induced diabetic rats. Eur J Nutr: July 30, 2018 (Epub ahead of print).

2. Wagnew F, Eshetie S, Kibret GD, Zegeye A, Dessie G, Mulugeta $\mathrm{H}$ and Alemu A: Diabetic nephropathy and hypertension in diabetes patients of sub-Saharan countries: A systematic review and meta-analysis. BMC Res Notes 11: 565, 2018.

3. Mafi A, Aghadavod E, Mirhosseini N, Mobini M and Asemi Z: The effects of expression of different microRNAs on insulin secretion and diabetic nephropathy progression. J Cell Physiol 234: 42-50, 2018.

4. Song X, Gong M, Chen Y, Liu H and Zhang J: Nine hub genes as the potential indicator for the clinical outcome of diabetic nephropathy. J Cell Physiol 234: 1461-1468, 2019.

5. Liu P, Peng L, Zhang H, Tang PM, Zhao T, Yan M, Zhao H, Huang X, Lan $\mathrm{H}$ and Li P: Tangshen formula attenuates diabetic nephropathy by promoting ABCA1-mediated renal cholesterol efflux in db/db Mice. Front Physiol 9: 343, 2018.

6. Wu T, Li Q, Wu T and Liu HY: Identification of biological targets of therapeutic intervention for diabetic nephropathy with bioinformatics approach. Exp Clin Endocrinol Diabetes 122: 587-591, 2014.

7. Ziyadeh FN and Wolf G: Pathogenesis of the podocytopathy and proteinuria in diabetic glomerulopathy. Curr Diabetes Rev 4: 39-45, 2008.

8. Wu J, Liu J, Ding Y, Zhu M, Lu K, Zhou J, Xie X, Xu Y, Shen X, Chen Y, et al: MiR-455-3p suppresses renal fibrosis through repression of ROCK2 expression in diabetic nephropathy. Biochem Biophys Res Commun 503: 977-983, 2018.

9. He F, Peng F, Xia X, Zhao C, Luo Q, Guan W, Li Z, Yu X and Huang F: MiR-135a promotes renal fibrosis in diabetic nephropathy by regulating TRPC1. Diabetologia 57: 1726-1736, 2014

10. Putta S, Lanting L, Sun G, Lawson G, Kato M and Natarajan R: Inhibiting MicroRNA-192 Ameliorates renal fibrosis in diabetic nephropathy. J Am Soc Nephrol 23: 458-469, 2012.

11. Chen Y, Qiao F, Zhao Y, Wang Y and Liu G: HMGB1 is activated in type 2 diabetes mellitus patients and in mesangial cells in response to high glucose. Int J Clin Exp Pathol 8: 6683-6691, 2015.

12. Seo E, Kang H, Oh YS and Jun HS: Psoralea corylifolia L. Seed extract attenuates diabetic nephropathy by inhibiting renal fibrosis and apoptosis in streptozotocin-induced diabetic mice. Nutrients 9: pii: E828, 2017.

13. Braga Gomes K, Fontana Rodrigues K and Fernandes AP: The role of transforming growth factor-beta in diabetic nephropathy. Int J Med Genet 2014: 1-6, 2014.

14. Hills CE and Squires PE: The role of TGF- $\beta$ and epithelial-to mesenchymal transition in diabetic nephropathy. Cytokine Growth Factor Rev 22: 131-139, 2011.

15. Cheng DW, Jiang Y, Shalev A, Kowluru R, Crook ED and Singh LP: An analysis of high glucose and glucosamine-induced gene expression and oxidative stress in renal mesangial cells. Arch Physiol Biochem 112: 189-218, 2006.

16. Chen Y, Teng L, Liu W, Cao Y, Ding D, Wang W, Chen H, Li C and An R: Identification of biological targets of therapeutic intervention for clear cell renal cell carcinoma based on bioinformatics approach. Cancer Cell Int 16: 16, 2016.

17. Tang Y, Zhang Z, Tang Y, Chen X and Zhou J: Identification of potential target genes in pancreatic ductal adenocarcinoma by bioinformatics analysis. Oncol Lett 16: 2453-2461, 2018.

18. Kanehisa M, Goto S, Sato Y, Furumichi M and Tanabe M: KEGG for integration and interpretation of large-scale molecular data sets. Nucleic Acids Res 40 (Database Issue): D109-D114, 2012. 
19. Szklarczyk D, Morris JH, Cook H, Kuhn M, Wyder S, Simonovic M, Santos A, Doncheva NT, Roth A, Bork P, et al: The STRING database in 2017: Quality-controlled protein-protein association networks, made broadly accessible. Nucleic Acids Res 45: D362-D368, 2017.

20. Wishart DS, Feunang YD, Guo AC, Lo EJ, Marcu A, Grant JR, Sajed T, Johnson D, Li C, Sayeeda Z, et al: DrugBank 5.0: A major update to the DrugBank database for 2018. Nucleic Acids Res 46: D1074-D1082, 2018.

21. Hsin KY, Matsuoka Y, Asai Y, Kamiyoshi K, Watanabe T, Kawaoka Y and Kitano H: SystemsDock: A web server for network pharmacology-based prediction and analysis. Nucleic Acids Res 44: W507-W513, 2016.

22. Hsin KY, Ghosh S and Kitano H: Combining machine learning systems and multiple docking simulation packages to improve docking prediction reliability for network pharmacology. PLoS One 8: e83922, 2013.

23. Ma J, Zhang L, Hao J, Li N, Tang J and Hao L: Up-regulation of microRNA-93 inhibits TGF- $\beta 1$-induced EMT and renal fibrogenesis by down-regulation of Orail. J Pharmacol Sci 136: 218-227, 2018.

24. Deshpande S, Abdollahi M, Wang M, Lanting L, Kato M and Natarajan R: Reduced autophagy by a microRNA-mediated signaling cascade in diabetes-induced renal glomerular hypertrophy. Sci Rep 8: 6954, 2018.

25. Song KH, Park J, Park JH, Natarajan R and Ha H: Fractalkine and its receptor mediate extracellular matrix accumulation in diabetic nephropathy in mice. Diabetologia 56: 1661-1669, 2013.

26. Kanwar YS, Sun L, Xie P, Liu FY and Chen S: A glimpse of various pathogenetic mechanisms of diabetic nephropathy. Annu Rev Pathol 6: 395-423, 2011

27. Reidy K, Kang HM, Hostetter T and Susztak K: Molecular mechanisms of diabetic kidney disease. J Clin Invest 124: 2333-2340, 2014.

28. Meng XM, Nikolic-Paterson DJ and Lan HY: TGF- $\beta$ : The master regulator of fibrosis. Nat Rev Nephrol 12: 325-328, 2016.

29. Lim AK and Tesch GH: Inflammation in diabetic nephropathy Mediators Inflamm 2012: 146154, 2012.

30. Lan HY: Transforming growth factor- $\beta /$ Smad signalling in diabetic nephropathy. Clin Exp Pharmacol Physiol 39: 731-738, 2012.

31. Xu L, Shen P,Bi Y, Chen J, Xiao Z, Zhang X and Wang Z: Danshen injection ameliorates STZ-induced diabetic nephropathy in association with suppression of oxidative stress, pro-inflammatory factors and fibrosis. Int Immunopharmacol 38: 385-394, 2016.

32. Jochum W, Passegué E and Wagner EF: AP-1 in mouse development and tumorigenesis. Oncogene 20: 2401-2412, 2001.

33. Weigert C, Sauer U, Brodbeck K, Pfeiffer A, Häring HU and Schleicher ED: AP-1 proteins mediate hyperglycemia-induced activation of the human TGF-beta1 promoter in mesangial cells J Am Soc Nephrol 11: 2007-2016, 2000.

34. Gao P, Wei Y, Zhang Z, Zeng W, Sun D, Liu D, Hou B, Zhang C, Zhang N, Li H and Li L: Synergistic effects of c-Jun and SP1 in the promotion of TGF $\beta 1$-mediated diabetic nephropathy progression. Exp Mol Pathol 100: 441-450, 2016.

35. Wang X, Yao B, Wang Y, Fan X, Wang S, Niu A, Yang H, Fogo A Zhang MZ and Harris RC: Macrophage Cyclooxygenase-2 protects against development of diabetic nephropathy. Diabetes 66: 494-504, 2017.

36. Watanabe $\mathrm{Y}$, Yamaguchi $\mathrm{T}$, Ishihara $\mathrm{N}$, Nakamura $\mathrm{S}$, Tanaka S, Oka R, Imamura H, Sato Y, Ban N, Kawana H, et al: 7-Ketocholesterol induces ROS-mediated mRNA expression of 12-lipoxygenase, cyclooxygenase-2 and pro-inflammatory cytokines in human mesangial cells: Potential role in diabetic nephropathy. Prostaglandins Other Lipid Mediat 134: 16-23, 2018.

37. Cheng HF, Wang CJ, Moeckel GW, Zhang MZ, McKanna JA and Harris RC: Cyclooxygenase-2 inhibitor blocks expression of mediators of renal injury in a model of diabetes and hypertension1. Kidney Int 62: 929-939, 2002.

38. Cheng H, Fan X, Moeckel GW and Harris RC: Podocyte COX-2 exacerbates diabetic nephropathy by increasing podocyte (pro) renin receptor expression. J Am Soc Nephrol 22: 1240-1251, 2011.
39. Ma X, Lu C, Lv C and Wang Q: The expression of miR-192 and its significance in diabetic nephropathy patients with different urine albumin creatinine ratio. J Diabetes Res 2016: 6789402, 2016.

40. Tian RQ, Wang XH, Hou LJ, Jia WH, Yang Q, Li YX, Liu M, Li X and Tang H: MicroRNA-372 is down-regulated and targets cyclin-dependent kinase 2 (CDK2) and cyclin A1 in human cervical cancer, which may contribute to tumorigene. J Biol Chem 286: 25556-25563, 2011.

41. Saurus P, Kuusela S, Dumont V, Lehtonen E, Fogarty CL, Lassenius MI, Forsblom C, Lehto M, Saleem MA, Groop PH and Lehtonen S: Cyclin-dependent kinase 2 protects podocytes from apoptosis. Sci Rep 6: 21664, 2016.

42. Huang K, Huang J, Chen C, Hao J, Wang S, Huang J, Liu P and Huang H: AP-1 regulates sphingosine kinase 1 expression in a positive feedback manner in glomerular mesangial cells exposed to high glucose. Cell Signal 26: 629-638, 2014.

43. Chen S, Mukherjee S, Chakraborty C and Chakrabarti S: High glucose-induced, endothelin-dependent fibronectin synthesis is mediated via NF-kappa B and AP-1. Am J Physiol Cell Physiol 284: C263-C272, 2003.

44. Haneda M, Koya D, Isono M and Kikkawa R: Overview of glucose signaling in mesangial cells in diabetic nephropathy. J Am Soc Nephrol 14: 1374-1382, 2003.

45. Cao AL, Wang L, Chen X, Wang YM, Guo HJ, Chu S, Liu C, Zhang XM and Peng W: Ursodeoxycholic acid and 4-phenylbutyrate prevent endoplasmic reticulum stress-induced podocyte apoptosis in diabetic nephropathy. Lab Invest 96: 610-622, 2016.

46. Chen Y, Gui D, Chen J,He D, Luo Y and Wang N: Down-regulation of PERK-ATF4-CHOP pathway by Astragaloside IV is associated with the inhibition of endoplasmic reticulum stress-induced podocyte apoptosis in diabetic rats. Cell Physiol Biochem 33: 1975-1987, 2014.

47. Lindenmeyer MT, Rastaldi MP, Ikehata M, Neusser MA, Kretzler M, Cohen CD and Schlöndorff D: Proteinuria and hyperglycemia induce endoplasmic reticulum stress. J Am Soc Nephrol 19: 2225-2236, 2008.

48. Chen J, Hou XF, Wang G, Zhong QX, Liu Y, Qiu HH, Yang N, Gu JF, Wang CF, Zhang L, et al: Terpene glycoside component from Moutan Cortex ameliorates diabetic nephropathy by regulating endoplasmic reticulum stress-related inflammatory responses. J Ethnopharmacol 193: 433-444, 2016.

49. Eletto D, Dersh D and Argon Y: GRP94 in ER quality control and stress responses. Semin Cell Dev Biol 21: 479-485, 2010.

50. Huang $M$ and Prendergast GC: RhoB in cancer suppression. Histol Histopathol 21: 213-218, 2006.

51. Bravo-Nuevo A, Sugimoto H, Iyer S, Fallon Z, Lucas JM, Kazerounian S, Prendergast GC, Kalluri R, Shapiro NI and Benjamin LE: RhoB loss prevents streptozotocin-induced diabetes and ameliorates diabetic complications in mice. Am J Pathol 178: 245-252, 2011.

52. Mehta G, Ferreira VP, Skerka C, Zipfel PF and Banda NK: New insights into disease-specific absence of complement factor $\mathrm{H}$ related protein $\mathrm{C}$ in mouse models of spontaneous autoimmune diseases. Mol Immunol 62: 235-248, 2014.

53. Bonomo JA, Palmer ND, Hicks PJ, Lea JP, Okusa MD, Langefeld CD, Bowden DW and Freedman BI: Complement factor $\mathrm{H}$ gene associations with end-stage kidney disease in African Americans. Nephrol Dial Transplant 29: 1409-1414, 2014.

54. Mallipattu SK, Estrada CC and He JC: The critical role of Krüppel-like factors in kidney disease. Am J Physiol Renal Physiol 312: F259-F265, 2017.

55. Guo Y, Pace J, Li Z, Ma'ayan A, Wang Z, Revelo MP, Chen E, Gu X, Attalah A, Yang Y, Estrada C, et al: Podocyte-specific induction of Krüppel-like factor 15 restores differentiation markers and attenuates kidney injury in proteinuric kidney disease. J Am Soc Nephrol 29: 2529-2545, 2018.

This work is licensed under a Creative Commons Attribution-NonCommercial-NoDerivatives 4.0 International (CC BY-NC-ND 4.0) License. 